\title{
Phase diagram of QCD in a magnetic background
}

\author{
Massimo D’Elia* and Lorenzo Maio $\oplus^{\dagger}$ \\ Dipartimento di Fisica dell'Università di Pisa and INFN-Sezione di Pisa, \\ Largo Pontecorvo 3, I-56127 Pisa, Italy \\ Francesco Sanfilippo \\ INFN-Sezione di Roma Tre, Via della Vasca Navale 84, I-00146 Rome, Italy \\ Alfredo Stanzione $\oplus^{\S}$ \\ SISSA, Via Bonomea 265, 34136, Trieste, Italy
}

(Received 2 December 2021; accepted 1 February 2022; published 23 February 2022)

\begin{abstract}
We provide numerical evidence that the thermal QCD crossover turns into a first order transition in the presence of large enough magnetic background fields. The critical end point is found to be located between $e B=4 \mathrm{GeV}^{2}$ [where the pseudocritical temperature is $T_{c}=(98 \pm 3) \mathrm{MeV}$ ] and $e B=9 \mathrm{GeV}^{2}$ [where the critical temperature is $\left.T_{c}=(63 \pm 5) \mathrm{MeV}\right]$. Results are based on the analysis of quark condensates and number susceptibilities, determined by lattice simulations of $N_{f}=2+1$ QCD at the physical point, discretized via rooted stout staggered fermions and a Symanzik tree level improved pure gauge action, adopting two different lattice spacings, $a=0.114$ and $0.086 \mathrm{fm}$, for $e B=9 \mathrm{GeV}^{2}$ and three, $a=0.114$, 0.086 , and $0.057 \mathrm{fm}$, for $e B=4 \mathrm{GeV}^{2}$. We also present preliminary results regarding the confining properties of the thermal theory, suggesting that they could change drastically going across the phase transition.
\end{abstract}

DOI: 10.1103/PhysRevD.105.034511

\section{INTRODUCTION}

The investigation of QCD properties in a magnetic background field has been the subject of various studies in the last few years, see, e.g., Refs. [1-3] for recent reviews. Part of the interest is directly related to phenomenology: strong background fields are expected in noncentral heavy ion collisions [4-9], in astrophysical objects like magnetars [10], and might have been produced during the cosmological electroweak phase transition [11,12], thus influencing the subsequent evolution of the Universe, including the cosmological QCD transition. Lattice QCD simulations have been essential to advance knowledge in this field, given also the fact that, unlike the case of a baryon chemical potential, no technical problem hinders the application of standard Monte Carlo techniques for the

\footnotetext{
*massimo.delia@unipi.it

lorenzo.maio@phd.unipi.it

francesco.sanfilippo@infn.it

§alfredo.stanzione@sissa.it
}

Published by the American Physical Society under the terms of the Creative Commons Attribution 4.0 International license. Further distribution of this work must maintain attribution to the author(s) and the published article's title, journal citation, and DOI. Funded by SCOAP ${ }^{3}$. computation of the QCD path-integral in a magnetic background.

One of the most relevant aspects regards the influence of the magnetic field on the QCD phase diagram. Early lattice studies of $N_{f}=2 \mathrm{QCD}$, adopting standard staggered fermions and heavier-than-physical quark masses, showed a slightly increasing behavior of the crossover temperature $T_{c}$ as a function of the magnetic field $B[13,14]$. That was however not confirmed by an investigation of $N_{f}=2+1$ QCD at the physical point discretized via improved staggered fermion, showing instead an appreciable decrease of $T_{c}$, of the order of 10-20\%, for magnetic fields going up to $e B \sim 1 \mathrm{GeV}^{2}$ [15], a behavior confirmed also by later lattice studies [16]. The reason for the discrepancy of early results has been clarified by later studies: it should be ascribed to lattice artifacts [17], while the decreasing behavior of $T_{c}$ as a function of $B$ is observed also for larger than physical pion masses $[18,19]$.

One important aspect, generally confirmed by lattice simulations, is the strengthening of the QCD crossover as the magnetic field is increased, which points to the possibility that it could turn into a real phase transition for large enough $B$. Available predictions, based on the extrapolation of lattice results and on the numerical study of effective models, suggest that this could happen for $e B$ of the order of $10 \mathrm{GeV}^{2}$ [20]. That could have significant 
implications for the physics of the early Universe, regarding in particular the consequences of a first order cosmological QCD transition [21,22]. As a matter of fact, a direct observation of this phenomenon has been reported only for discretizations adopting unimproved staggered quarks [17], for which however one also observes that $T_{c}$ increases (instead of decreasing) with $B$.

The main purpose of the present study is to push forward our knowledge on this topic, by exploring finite temperature $N_{f}=2+1$ QCD with physical quark masses at unprecedented values of the magnetic field, trying also to keep control on UV cutoff effects. In order to do that, we will consider a stout improved staggered discretization of the theory and two different values of the magnetic field, $e B \simeq 4$ and $9 \mathrm{GeV}^{2}$, trying to keep control on discretization effects by exploring two different lattice spacings, $a=0.057$ and $0.086 \mathrm{fm}$, for $e B=9 \mathrm{GeV}^{2}$, and three, $a=0.057,0.086$, and $0.114 \mathrm{fm}$, for $e B=4 \mathrm{GeV}^{2}$. Anticipating part of the final results, we will provide evidence that the QCD transition is first order for $e B=$ $9 \mathrm{GeV}^{2}$ and that the critical temperature, for that value of the magnetic field, goes down to values below $70 \mathrm{MeV}$. We will also present a preliminary investigation of the confining properties of the theory, suggesting that they could change drastically going across the phase transition.

Even if our investigation is not affected by any technical obstruction, such as a sign problem, it is anyway extremely challenging from a numerical point of view. On one hand, the need for large magnetic fields requires correspondingly fine lattice spacings of the order or below $0.1 \mathrm{fm}$. On the other hand, given the fact that the critical temperature keeps its steady decrease with $B$, we require simulations with an Euclidean time compactification length around $2 \mathrm{fm}$ or larger, meaning that, in order to reach lattice spacings below $0.1 \mathrm{fm}$, we need to perform simulations on lattices with a large number of sites in the temporal direction (a few tens). That sets by itself a strong limitation to the explorable lattices, in particular regarding the aspect ratios (ratio of the spatial to the Euclidean time lattice extents), which are reasonably affordable, given the available computational resources. ${ }^{1}$ In particular regarding the approach to the thermodynamical limit, our results should be considered as exploratory, but nevertheless providing an already consistent and clear picture, which claims for future investigations and refinements.

The paper is organized as follows. In Sec. II we discuss the lattice discretization of the theory and other technical details regarding the implementation of the magnetic background field and the physical observables explored in our investigation. In Sec. III we present and discuss our

\footnotetext{
${ }^{1}$ In particular, we were not able, with the available lattice sizes, to provide results at the finest lattice spacing, $a=0.057 \mathrm{fm}$, for $e B=9 \mathrm{GeV}^{2}$.
}

numerical results. Finally, in Sec. IV we draw our conclusions and discuss future perspectives.

\section{NUMERICAL METHODS}

As in Refs. [18,23], we consider a discretization of $N_{f}=2+1$ QCD based on the tree-level improved Symanzik pure gauge action $[24,25]$ and on stout rooted staggered fermions [26,27], i.e., on the following partition function

$$
Z=\int[D U] e^{-S_{\mathrm{YM}}} \prod_{f=u, d, s} \operatorname{det}\left(M_{s t}^{f}\right)^{\frac{1}{4}}
$$

where $[D U]$ is the Haar measure for gauge links, $f$ is the flavor index, and the fermion matrix and the gauge action are, respectively,

$$
\begin{aligned}
M_{s t i j}^{f} & =\hat{m}_{f} \delta_{i j}+\sum_{\nu=1}^{4} \frac{\eta_{i ; \nu}}{2}\left(U_{i ; \nu}^{(2)} \delta_{i j-\hat{\nu}}-U_{i-\hat{\nu} ; j}^{(2) \dagger} \delta_{i j+\hat{\nu}}\right), \\
S_{\mathrm{YM}} & =-\frac{\beta}{3} \sum_{\substack{i \\
\mu \neq \nu}}\left(\frac{5}{6} W_{i, \mu \nu}^{1 \times 1}-\frac{1}{12} W_{i, \mu \nu}^{1 \times 2}\right),
\end{aligned}
$$

with periodic (antiperiodic) boundary conditions in the Euclidean temporal direction for bosonic (fermionic) fields, in order to reproduce thermal conditions. There, $i, j$ and $\mu$, $\nu$ are, respectively, lattice sites and directions, while $\beta$ is the inverse gauge coupling, $a$ is the lattice spacing, and $\hat{m}_{f}=a m_{f}$ are the dimensionless bare quark masses. The $\eta_{i ; \nu}$ are the staggered quark phases, $U_{i ; \nu}^{(2)}$ is the two-times stout smeared link (with isotropic smearing parameter $\rho=0.15$ ), while $W_{i, \mu \nu}^{1 \times}$ s are the real parts of the trace of the link products along the $1 \times 1$ and $1 \times 2$ rectangular closed path, respectively.

Bare quark masses and the gauge coupling values have been tuned in order to move on a line of constant physics, which reproduces experimental results for hadronic observables, based on the determinations reported in Refs. [2830]. In particular, as in Ref. [23], we have considered three different lattice spacings, $a \simeq 0.057,0.086$, and $0.114 \mathrm{fm}$; for each lattice spacing the physical temperature of the system, which is equal to the inverse of the Euclidean temporal extension, $T=1 /\left(N_{t} a\right)$, has been tuned by changing the number of temporal lattice sites $N_{t}$ at fixed $a$. Such a fixed scale approach to thermodynamics has the drawback of not allowing for a fine tuning of the physical temperature; however, it has many advantages at the same time, since it simplifies both the renormalization of physical observable and the continuum extrapolation at fixed physical values of the external background field, as we discuss in the following. 


\section{A. External magnetic field}

In the lattice approach, the presence of an external magnetic background field can be translated in the introduction of additional $U(1)$ phases to the elementary parallel transporters

$$
U_{i ; \mu}^{(2)} \rightarrow u_{i ; \mu}^{f} U_{i ; \mu}^{(2)},
$$

which are kept constant, i.e., no functional integration is performed over them, and are different for the different flavors, depending on their electric charge. In particular, considering a uniform magnetic field $\vec{B}$ in the $\hat{z}$ direction and the following gauge choice

$$
A_{t}=A_{x}=A_{z}=0, \quad A_{y}(x)=B x,
$$

a possible discretization on a periodic toroidal lattice is the following

$$
u_{i ; y}^{f}=e^{i a^{2} q_{f} B i_{x}},\left.\quad u_{i ; x}^{f}\right|_{i_{x}=L_{x}}=e^{-i a^{2} q_{f} L_{x} B i_{y}},
$$

with all other $U(1)$ link variables set to one, where $L_{i}$ is the number of lattice sites along direction $i$ and last condition guarantees smoothness of the magnetic field across the $x$ boundary [31-33]. This choice leads to a constant magnetic field but for a single plaquette, which is pierced by an additional Dirac string and guarantees a zero magnetic flux across the lattice torus; invisibility of that string leads to a quantization condition for $B$, which is more compelling for the smallest quark charge $q_{f}=e / 3$ :

$$
q_{f} B=\frac{2 \pi b_{z}}{a^{2} L_{x} L_{y}} \Rightarrow e B=\frac{6 \pi b_{z}}{a^{2} L_{x} L_{y}}, \quad b_{z} \in \mathbb{Z} .
$$

The external field leads to additional discretization errors. Since the magnetic field acts on the system through the gauge invariant $U(1)$ phase factors that dynamical quarks pick up going through closed loops on the lattice, the phase factor for the smallest nontrivial loop (a plaquette in the $x y$ plane)

$$
\exp \left(i q_{f} B a^{2}\right)=\exp \left(i \frac{6 \pi b_{z}}{L_{x} L_{y}} \frac{q_{f}}{e}\right)
$$

must be much smaller than $2 \pi$, hence

$$
\frac{2 b_{z}}{L_{x} L_{y}} \ll 1
$$

where we have considered the up quark, for which discretization errors are larger; all that sets a UV cutoff for the largest field explorable for a given lattice spacing, $e B \leq 2 \pi / a^{2}$. For the coarsest lattice studied in this study, $a \simeq 0.114 \mathrm{fm}$, the cutoff is around $20 \mathrm{GeV}^{2}$, which is not too far from $e B=9 \mathrm{GeV}^{2}$ : this is at the origin of sizable discretization effects observed for this value of the magnetic field, which disappear only after a proper continuum extrapolation [23].

\section{B. Observables}

The determination of the (pseudo)critical temperature $T_{c}$ will be based on the analysis of the renormalized chiral condensate and of the susceptibility of the strange quark number, which are two standard observables used for the same purposes in previous studies.

The $f$-flavor condensate is defined as

$\langle\bar{\psi} \psi\rangle_{f}=\frac{\partial}{\partial m_{f}}\left(\frac{T}{V_{s}} \log Z\right)=\frac{1}{4 a^{3} L_{s}^{3} N_{t}}\left\langle\operatorname{Tr}\left(M_{s t}^{f}\right)^{-1}\right\rangle$,

where $V_{s}$ is the spatial volume and the trace of the inverse fermion matrix is determined configuration by configuration, as usual, by means of noisy estimators. The condensate is affected by both additive and multiplicative renormalizations, which can be subtracted following the prescription of Ref. [34]

$\langle\bar{\psi} \psi\rangle_{f}^{r}(B, T)=\frac{m_{f}}{m_{\pi}^{2} F_{\pi}^{2}}\left(\langle\bar{\psi} \psi\rangle_{f}(B, T)-\langle\bar{\psi} \psi\rangle_{f}(0,0)\right)$.

The zero- $T$ subtraction, which is performed at fixed UV cutoff, eliminates additive divergences, while multiplication by the bare quark mass $m_{f}$ takes care of multiplicative ones.

In the following we will show results for the sum of up and down contributions, i.e., the renormalized light quark condensate $\Sigma_{l}^{r}(B, T)$. The behavior of $\Sigma_{l}^{r}(B, T)$ will be monitored to locate $T_{c}$, looking for its inflection point in the region where it drops towards zero. Just for the purpose of a finite size scaling analysis around the transition, we will consider also the unrenormalized disconnected chiral susceptibility

$$
\chi_{\bar{\psi} \psi \mu, f}^{\mathrm{disc}} \equiv \frac{1}{16 L_{s}^{3} N_{t}}\left[\left\langle\left(\operatorname{Tr} M_{f}^{-1}\right)^{2}\right\rangle-\left\langle\operatorname{Tr} M_{f}^{-1}\right\rangle^{2}\right]
$$

The dimensionless susceptibility of the strange quark number is instead defined as follows (with $f=s$ ):

$$
\begin{aligned}
\chi_{f} & \equiv \frac{1}{T^{2}} \frac{\partial}{\partial \mu_{f}^{2}}\left(\frac{T}{V_{s}} \log Z\right), \\
& =\frac{N_{t}}{4 L_{s}^{3}}\left\langle\operatorname{Tr}\left[M_{f}^{-1} \partial_{a \mu_{f}}^{2} M_{f}-\left(M_{f}^{-1} \partial_{a \mu_{f}} M_{f}\right)^{2}\right]\right\rangle,
\end{aligned}
$$

where $\mu_{f}$ is the quark chemical potential and, in the last line, only terms which do not vanish at $\mu_{f}=0$ have been left in. ${ }^{2}$

\footnotetext{
${ }^{2}$ Considering the standard introduction of the chemical potential on the lattice, where temporal gauge links in the forward/ backward temporal direction get multiplied by a factor $\exp \left( \pm a \mu_{f}\right)$, the derivatives $\partial_{a \mu_{f}} M_{f}$ and $\partial_{a \mu_{f}}^{2} M_{f}$ correspond to just the temporal part of the Dirac operator, with an additional minus for each derivative in the backward propagation.
} 


\section{NUMERICAL RESULTS}

Most of our simulations have been carried out at three different lattice spacings, $a \simeq 0.057,0.086$, and $0.114 \mathrm{fm}$, keeping the spatial size fixed at $a L_{s} \simeq 2.75 \mathrm{fm}$ and varying the temporal lattice size $N_{t}$ in order to change the temperature. For reasons to be discussed below, results at the finest lattice spacing are only available for $e B=4 \mathrm{GeV}^{2}$. In this setup the magnetic field, according to Eq. (6), is kept fixed in physical units by just using the same number of quanta $b_{z}$ for every lattice spacing: that makes the continuum extrapolation much easier.

In particular, we have fixed $b_{z}=41$ and $b_{z}=93$, respectively, for 4 and $9 \mathrm{GeV}^{2}$. Larger spatial sizes, up to $\sim 4 \mathrm{fm}$, have been explored in a few cases, in order to check the impact of finite size effects, or to perform a finite size scaling analysis around the transition: in those cases, $b_{z}$ has been increased accordingly in order to keep $e B$ fixed, see Eq. (6). Additional simulations, needed for zero temperature subtractions or normalization, have been performed for $e B=0,4$, and $9 \mathrm{GeV}^{2}$ on lattices with a temporal extension of around $5.5 \mathrm{fm}$, which is large enough to be considered as a good approximation for $T \simeq 0 .{ }^{3}$

Monte Carlo sampling of gauge configurations has been performed based on a rational hybrid Monte Carlo (RHMC) algorithm running on GPUs $[35,36]$. For each simulation we performed $O\left(10^{3}\right)$ RHMC trajectories of unit length, taking measures every five trajectories.

\section{A. The finite temperature transition at large $e B$}

In Fig. 1 we show the renormalized light condensate $\Sigma_{l}^{r}(B, T)$ as a function of $T$ for the two explored values of $e B$ and for various lattice spacings and spatial extensions. Results have been normalized by those obtained for the same values of $B$ at $T \simeq 0$ : that suppresses much of the UV cutoff dependence already observed for $e B=9 \mathrm{GeV}^{2}$ at $T=0$ in Ref. [23].

A residual UV cutoff, as well as a finite size dependence, is visible around the transition; however that does not obscure the main message emerging from Fig. 1. $T_{c}$ is around $100 \mathrm{MeV}$ for $e B=4 \mathrm{GeV}^{2}$ and drops below $80 \mathrm{MeV}$ for $e B=9 \mathrm{GeV}^{2}$. Moreover, one observes a significant strengthening of the transition, which seems to become strong first order, with a large gap in the chiral condensate, at the larger value of $e B$.

Some considerations should be made about the possible weaknesses of our results. We have been forced to work with aspect ratios $L_{s} / N_{t}$ around 2, which is marginally compatible with a reliable study of thermodynamics, by

\footnotetext{
${ }^{3}$ Given the relatively low temperatures explored in this study, this is not a trivial statement. Actually, our reference "zero temperature" lattice corresponds to $T \simeq 36 \mathrm{MeV}$, which is well below the explored values of $T$ and deep in the confined region, at least for the present values of $e B$.
}
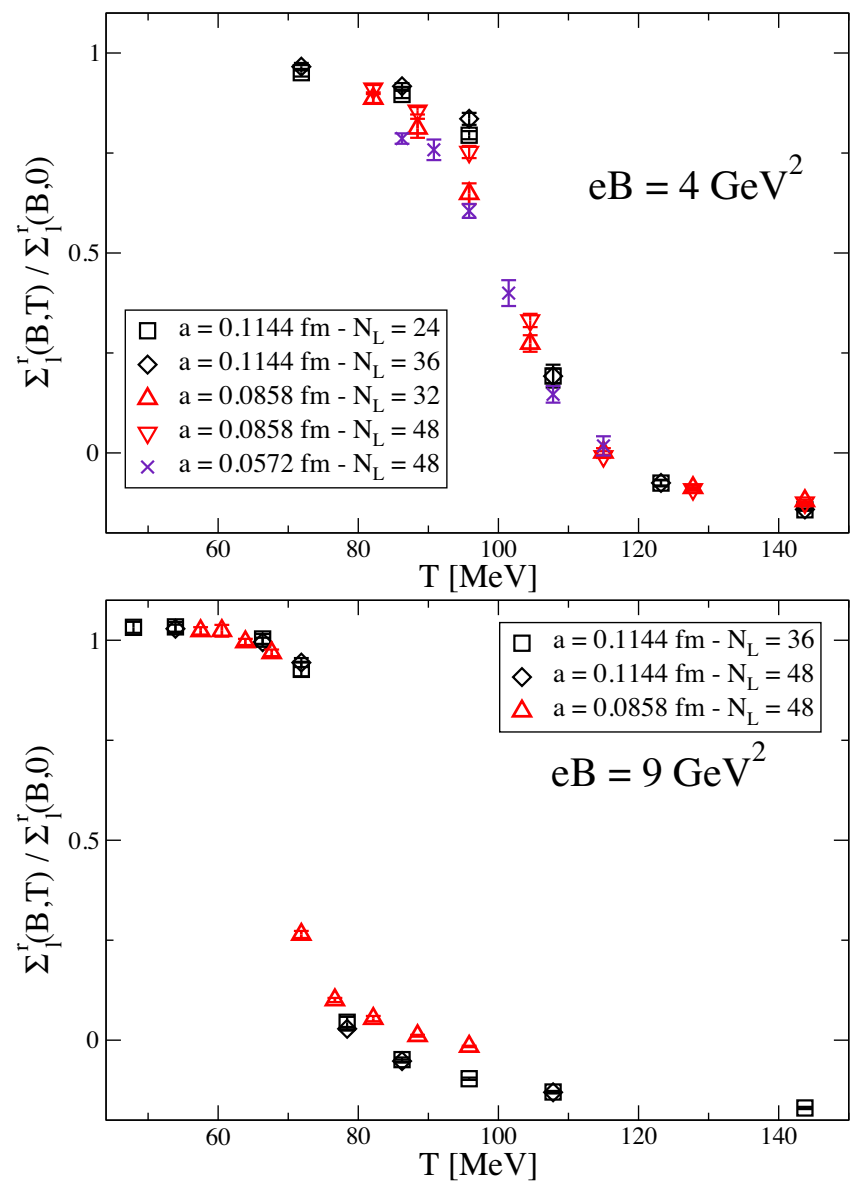

FIG. 1. Renormalized chiral condensates, divided by their values at $T=0$, obtained for $e B=4 \mathrm{GeV}^{2}$ (top) and $e B=$ $9 \mathrm{GeV}^{2}$ (bottom) for various lattice spacings and spatial extensions. The drop of the pseudocritical temperature is clearly visible, as well as the appearance of a well defined gap at the larger value of $e B$.

some converging constraints: the fact that the range of physically relevant temperatures turns out to be lower than expected from previous lattice studies [20], and the need for lattice spacings fine enough to support the explored values of $e B$, all that combined with a limited budget of available computational resources. This is also the reason we do not have results available for the finest lattice spacing at $e B=9 \mathrm{GeV}^{2}$, since in that case, without a significant increase of $L_{s}$, the aspect ratio would have been close to 1 around the transition.

Nevertheless, the main results depicted above do not seem to be much affected by such weaknesses. The dependence on the finite spatial size is visible around the transition but is not significant. The value of $T_{c}$ at $e B=9 \mathrm{GeV}^{2}$, where only two lattice spacings are available, seems to decrease even more when moving from the coarser to the finer lattice, while the transition is sharp and seemingly strong first order in both cases. 


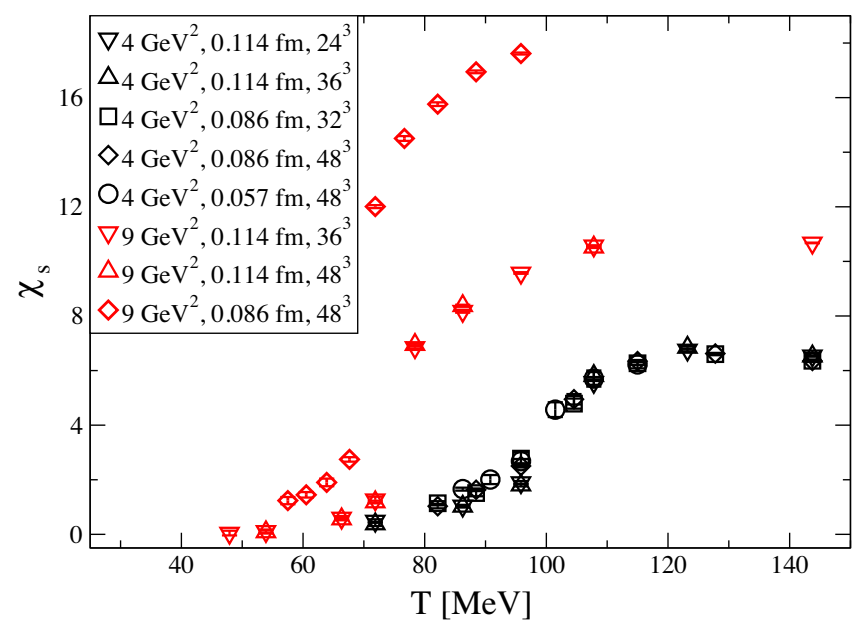

FIG. 2. Strange quark number susceptibility as a function of $T$ for both explored values of $e B$ and for various lattice spacings and spatial extensions. Significant UV cutoff effects are visible for the larger magnetic field, however this does not affect the conclusion for the appearance of a large gap at the transition in that case.

Similar conclusions are obtained by looking at results for the strange quark susceptibility, which are reported in Fig. 2. The susceptibility raises in correspondence of the same temperatures at which the chiral condensate drops, and has a sudden jump, suggesting a strong first order transition, for $e B=9 \mathrm{GeV}^{2}$. In the latter case, UV cutoff effects are clearly visible and significant, even if they affect mostly the overall magnitude of the susceptibility, and only marginally the location of $T_{c}$ : similar significant UV cutoff effects have been reported in Ref. [23], at the same value of $e B$, for the chiral condensate, and can be ascribed to the rough discretization of such large magnetic field, since up quarks pick an elementary phase around plaquettes which is large $(\sim 2 \pi / 3$ and $\sim \pi / 3$, respectively, for $a=0.114 \mathrm{fm}$ and $a=0.086 \mathrm{fm})$. We notice that the magnetic field induces a strong enhancement in quark number susceptibilities: similar observations have been reported in Ref. [37].

Results obtained for $T_{c}$ from both observables are shown as a function of $a^{2}$ in Fig. 3. For $e B=4 \mathrm{GeV}^{2} T_{c}$ has been determined by fitting the inflection point of $\Sigma_{l}^{r}$ or $\chi_{s}$, while for $e B=9 \mathrm{GeV}^{2}$ the determination coincides with the midpoint of the two temperatures where the sharp jump is observed, with an uncertainty given by their halfdifference; a systematic uncertainty of around $2 \%$, related to the determination the lattice spacing [28-30], should be considered in both cases. A tentative continuum extrapolation of $T_{c}$, assuming $O\left(a^{2}\right)$ corrections, is also reported for $e B=4 \mathrm{GeV}^{2}$, leading to $T_{c}\left(e B=4 \mathrm{GeV}^{2}\right)=$ $(98 \pm 3) \mathrm{MeV}$, while for $e B=9 \mathrm{GeV}^{2}$ we do not have enough degrees of freedom even for a linear fit. In the latter case, given the two available lattice spacings and all other systematic uncertainties, we believe that a safe and

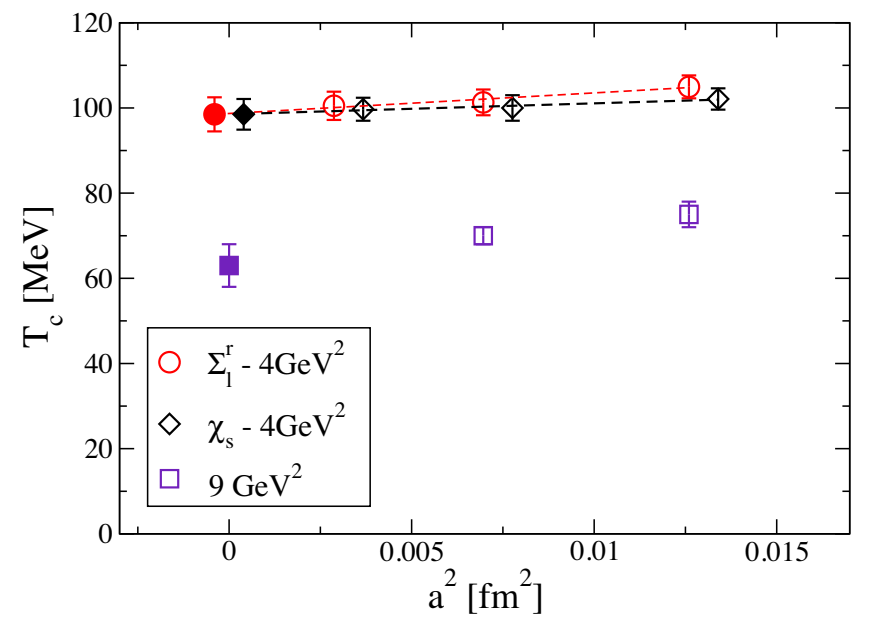

FIG. 3. Transition temperatures as a function of $a^{2}$, determined from the chiral condensate and from the strange quark number susceptibility at the two explored values of $e B$. For $e B=4 \mathrm{GeV}^{2}, T_{c}$ has been determined by fitting the inflection point of $\Sigma_{l}^{r}$ or $\chi_{s}$. For $e B=9 \mathrm{GeV}^{2}$, instead, the determination is obtained from the sharp jump observed for both quantities, with an uncertainty given by the half-difference of the temperatures on the two sides of the jump. A tentative continuum extrapolation of $T_{c}$, assuming $O\left(a^{2}\right)$ corrections, is reported in both cases, however for $e B=9 \mathrm{GeV}^{2}$ this is not even a fit, since only two lattice spacings are available.

conservative estimate for the continuum extrapolated temperature is $T_{c}\left(e B=9 \mathrm{GeV}^{2}\right)=(63 \pm 5) \mathrm{MeV}$.

We have put the two critical temperatures, together with previous results available in the literature, in order to draw a first tentative sketch of the updated QCD phase diagram in a magnetic field, which is reported in Fig. 4. A first observation is that our results, which are consistent with all previous direct lattice determinations, point to a steady decrease of $T_{c}$ even in the large field region, contrary to a much smoother approach to the infinite $B$ limit reported in the investigation of Ref. [20], which however was based on an effective description of QCD at large $e B$ in terms of an anisotropic pure gauge theory $[3,38]$. The second observation is that our results strongly suggest the presence of a strong first order transition, with a critical end point along the line which continuously connect $T_{c}(e B=0)$ with $T_{c}\left(e B=9 \mathrm{GeV}^{2}\right)$. The presence of a first order transition at large $e B$ was predicted in previous literature, with an estimate for the critical end point, based on an extrapolation, at $e B_{c}=10(2) \mathrm{GeV}^{2}$ [20]; our results suggest, for the first time from a direct lattice determination, that the critical point is located somewhere in the middle between $e B=4$ and $9 \mathrm{GeV}^{2}$. Such a conclusion however requires some deepening of our investigation, based on a finite size scaling analysis, in order to assess that at $e B=9 \mathrm{GeV}^{2}$ the transition is indeed first order: this is done in the following subsection. 


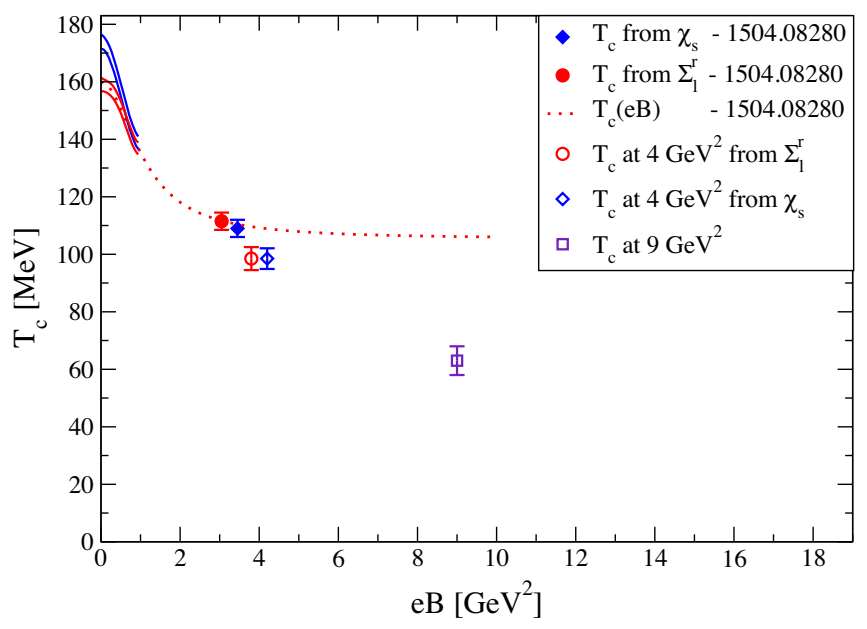

FIG. 4. We draw a first sketch of the updated version of the QCD phase diagram in a magnetic field, where our continuum extrapolated determinations of $T_{c}$ at $e B=4$ and $9 \mathrm{GeV}^{2}$ are plotted together with previous lattice determinations as well as tentative extrapolations (dotted line). The blue and red bands in the small field region are continuum extrapolations from Ref. [15], obtained, respectively, from the strange quark number susceptibility and the quark condensate. The determinations of Ref. [20] are not extrapolated to the continuum limit, which may account for their values seeming a bit higher.

\section{B. Finite size scaling around the critical temperature}

The fixed UV cutoff approach we have followed till now allows only for a discrete set of temperatures; as a consequence, a large jump in some observables somewhere is only suggestive of a first order transition but does not necessarily imply it. Smoking guns would be instead the presence of metastable histories, double peak distributions, and a proper finite size scaling (FSS) analysis, which however require a fine tuning of the temperature around the transition point.

Therefore, in order to clarify this aspect, we have decided to give up our fixed cutoff approach for a set of dedicated simulations. In particular, we have chosen one of the two simulation points at $e B=9 \mathrm{GeV}^{2}$ adjacent to the jump, taking it as the starting point for a temperature scan where $N_{t}$ is kept fixed and $T$ is changed by tuning the lattice spacing through the bare parameters.

As a further variation, the lattice spacing has been changed by tuning just the inverse gauge coupling $\beta$, which enters the pure gauge action, and not the bare quark masses, which enter the fermion determinant. That simplifies the FSS analysis, allowing for an easy application of the multihistogram method, in particular without the need for a costly reweighting of the fermion determinant. On the other hand, that has the drawback of moving us away from the physical line; however it should be clear that this is not a relevant aspect: we are just doing a fine-tuning, with the purpose of crossing the critical surface somewhere close to the starting point and test if it is first order or not. Since the presence of a first order transition, i.e., of a gap in physical observables, is stable under small variations of the parameters, we will obtain a valid and clear-cut answer anyway.

In order to make the computational effort of the FSS analysis affordable, we worked on the coarsest lattice; on the other hand, the jumps observed in the chiral condensate and in the strange quark number susceptibility suggest that the transition does not weaken going towards the continuum limit. As a starting point, we have chosen the $N_{t}=22$ lattice at $\beta=3.787, a m_{s}=0.0457$, and $a m_{u / d}=$ 0.00162 , which corresponds to $T \simeq 78.5 \mathrm{MeV}$ and is the first point on the upper side of the transition, and changed $\beta$ downwards, so as to increase the lattice spacing and decrease $T$, till we have crossed the transition. This has been repeated for three different spatial sizes, $L_{s}=24,30$, and 36.

As a first result, in Fig. 5 we show the disconnected and unrenormalized $^{4}$ chiral susceptibility $\chi_{\text {disc }, u}$ of the up quark (similar results are obtained for the down quark). Results clearly show that the susceptibility increases with the volume and that data collapse onto each other according to the following FSS ansatz ( $\phi$ is an unknown scaling function)

$$
\frac{\chi_{\mathrm{disc}, u}\left(L_{s}, \beta\right)}{L_{s}^{\gamma / \nu}}=\phi\left(\left(\beta-\beta_{c}\right) L_{s}^{1 / \nu}\right),
$$

when $\nu$ and $\gamma$ are fixed to the expected effective first order critical indexes for three spatial dimensions, i.e., $\nu=1 / 3$ and $\gamma=1$. The critical value of $\beta$ in Fig. 5, which optimizes the collapse, is $\beta_{c} \simeq 3.780$.

As further evidence of the presence of a first transition, now we focus on Monte Carlo (MC) histories of some observables, looking for the presence of double peak distributions or metastable behaviors around the transition. Figure 6 shows the MC history of the light chiral condensate, in units of HMC trajectories of unit length, on the $L_{s}=24$ lattice at $\beta=3.7755$ : the history clearly oscillates between two values, with a corresponding and well-defined double peak distribution.

As we move to a larger lattice, $L_{s}=36$, the double peak distribution becomes so sharp that the system is not able to easily tunnel from one phase to the other in a reasonable MC time. This is clear from Fig. 7, where we show the MC histories of two twin runs, performed with exactly the same parameters but starting from different sides of the phase transition: the two runs keep staying in their phase for a few

\footnotetext{
${ }^{4}$ Since we want to explore the critical behavior of the chiral susceptibility as the thermodynamical limit is approached, looking at just the disconnected part, which is expected to diverge itself at a genuine transition, is enough. For the same reason, the subtraction of regular (at fixed UV cutoff) renormalization constants is irrelevant to our purposes.
} 

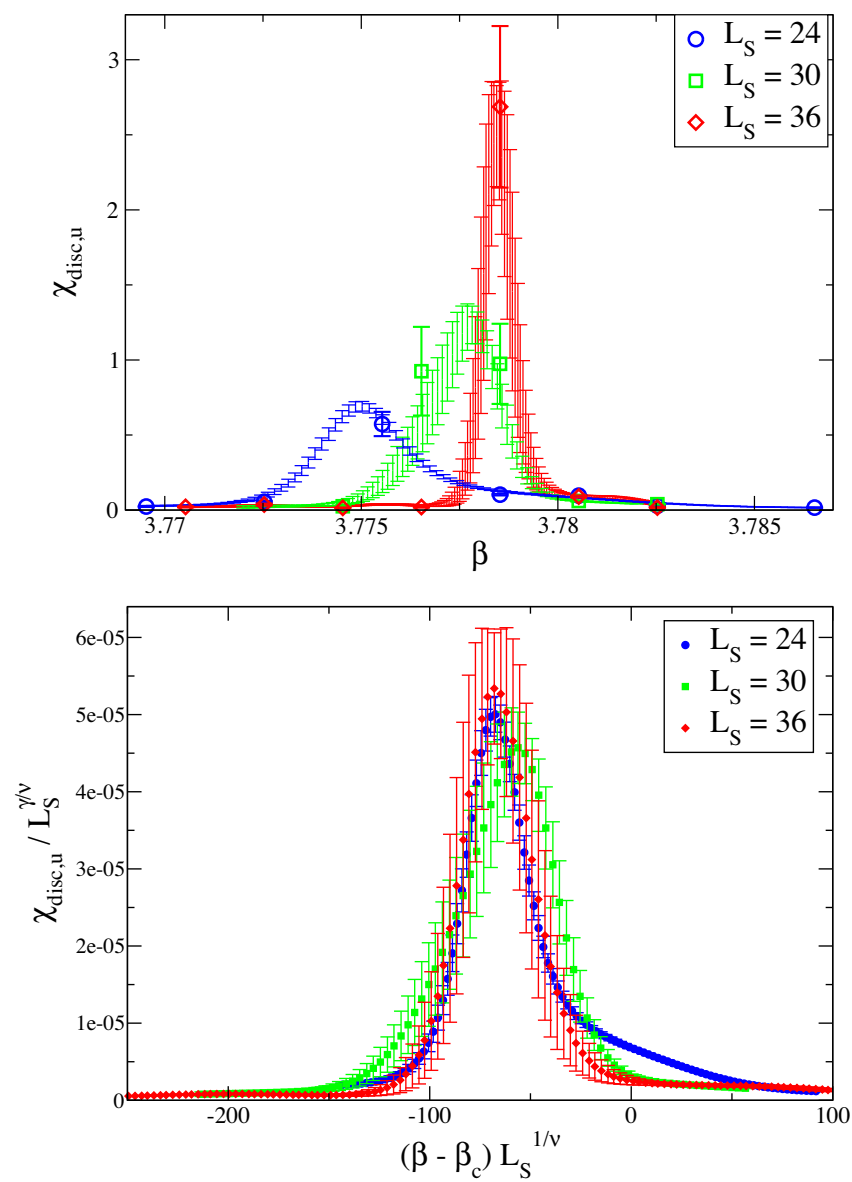

FIG. 5. FSS analysis of the unrenormalized disconnected chiral susceptibility of the up quark. Data have been obtained on lattices with $N_{t}=22$, fixing $b_{z}=93,145,209$, respectively, for $L_{s}=24,30,36$ so as to keep $e B$ constant as the thermodynamical limit is approached; the inverse gauge coupling $\beta$ has been tuned while keeping the bare quark masses fixed at $a m_{s}=$ 0.0457 and $a m_{u / d}=0.00162$. The FSS ansatz has been checked (lower figure) by fixing $\nu=1 / 3$ and $\gamma=1$, as expected around a first order transition, with $\beta_{c} \simeq 3.780$.

thousands of RHMC trajectories; moreover, in this case the bistability is clearly visible also in the pure gauge action.

\section{Confining properties of the two phases}

Having clarified that the large $B$ region of the $B-T$ phase diagram is characterized by a well-defined phase separation, a number of interesting questions emerge, regarding the properties and differences between the two phases. It is not the purpose of the present investigation to give a comprehensive answer to such questions, however we would like to touch at least one aspect, which has been already considered in some previous studies and regards the confining properties of the theory [23,39-44].

It is known that, at zero temperature, the static quarkantiquark potential become anisotropic, with a suppression of the string tension in the direction parallel to the magnetic

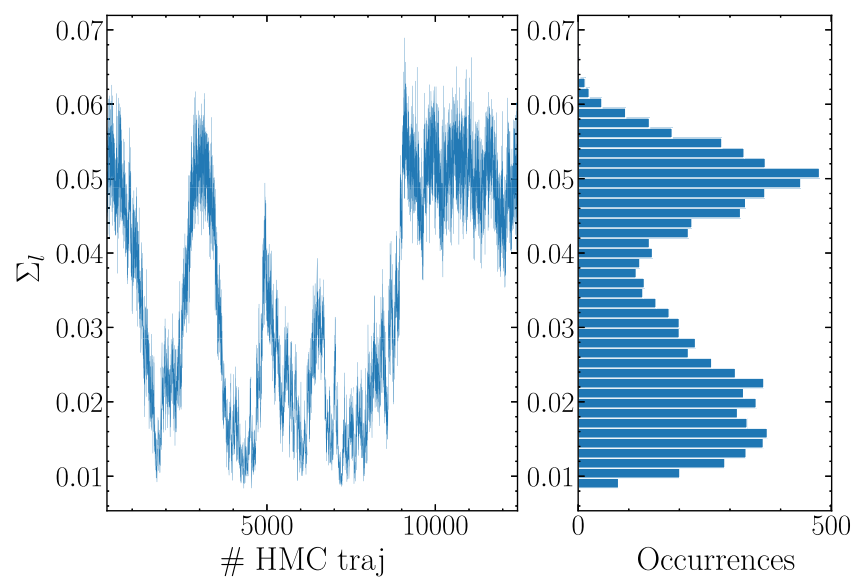

FIG. 6. MC history and distribution of the light quark condensate on the $24^{3} \times 22$ lattice at $\beta=3.7755, a m_{s}=0.0457$, $a m_{u / d}=0.00162$, and $b_{z}=93$. The bistability and the corresponding double peak distribution are clearly visible.
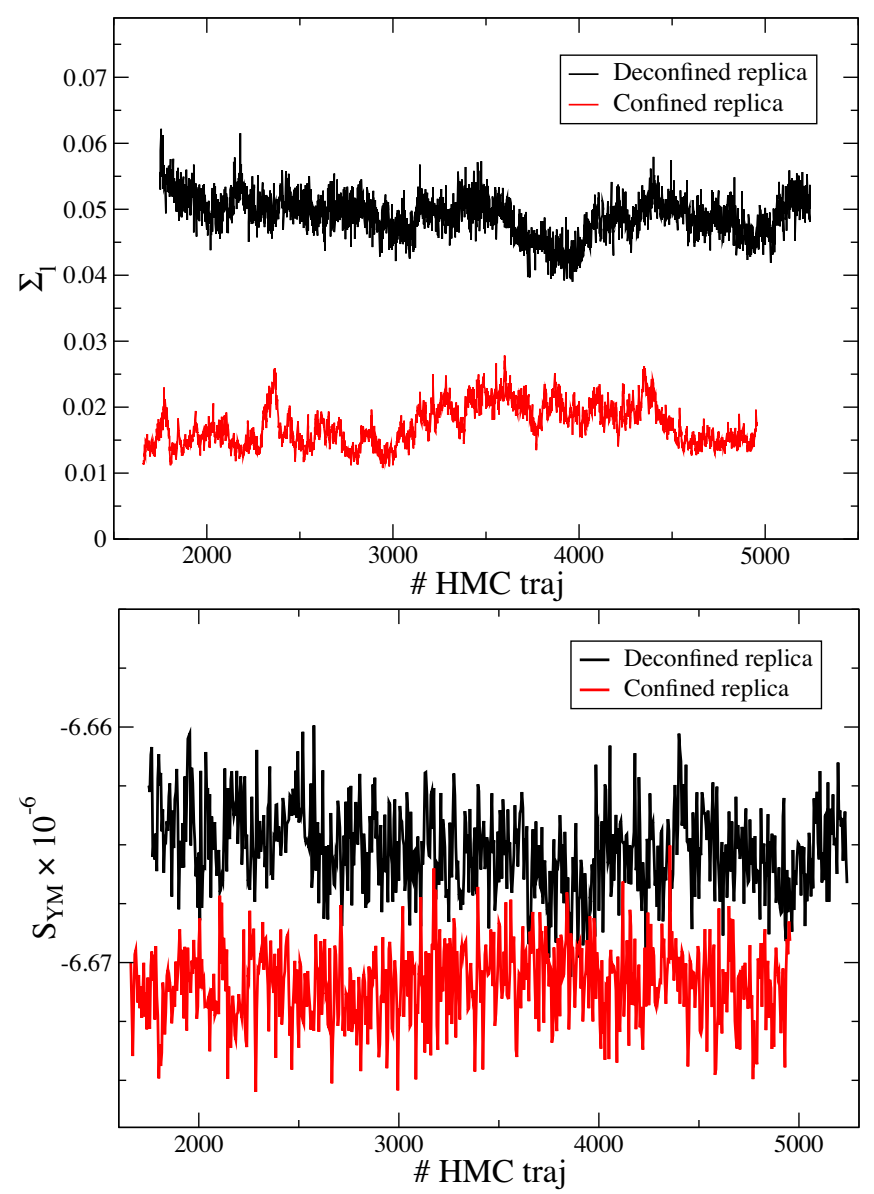

FIG. 7. Two twin MC histories obtained on the $36^{3} \times 22$ lattice at $\beta=3.7785, a m_{s}=0.0457, a m_{u / d}=0.00162$, and $b_{z}=209$. The two runs have been started from different sides of the phase transition, and keep staying in their starting phase for the whole run, consisting of a few thousands of RHMC trajectories of unit length. We show both the light quark condensate (top) and the pure gauge action (down). 
field, and an enhancement in the transverse directions [23,40-43]. The longitudinal string tension $\sigma_{L}$ is suppressed by more than one order of magnitude at $e B=9 \mathrm{GeV}^{2}$, with respect to its value at $e B=0$, while the transverse string tension $\sigma_{T}$ seems to saturate its increase at a value which is around $50 \%$ higher that the $B=0$ value [23]. The possible existence of a critical magnetic field $B_{c}$ at $T=0$, where the longitudinal string tension vanishes, and what could happen at such a critical field, is still unclear [23]. On the other hand, studies at finite temperature and up to moderate values of the magnetic field $[42,44]$ have shown that anisotropies in the static potential become less significant approaching the phase transition.

As a minimal, additional contribution to the investigation of the confining properties in the $B-T$ plane, we decided to investigate the static quark-antiquark potential at a fixed value of the temperature, $T \simeq 86 \mathrm{MeV}$, for the two different explored magnetic fields, $e B=4$ and $9 \mathrm{GeV}^{2}$. According to Fig. 4, the two simulations points should lay on the two different sides of the transition line. In this case we have decided to perform the investigation on the finest lattice, whose size is $48^{3} \times 40$.

In order to determine the static quark-antiquark potential, similarly to Refs. [23,41,42], we studied the Wilson loop $\left\langle\operatorname{Tr} W\left(a \vec{n}, a n_{t}\right)\right\rangle$ and its dependence on the Euclidean time $a n_{t}$, exploiting the relation

$$
\left\langle\operatorname{Tr} W\left(a \vec{n}, a n_{t}\right)\right\rangle \propto e^{-a V(a \vec{n}) n_{t}},
$$

which holds for large enough $a n_{t}$. In particular, from the previous equation one can derive

$$
a V(a \vec{n})=\lim _{n_{t} \rightarrow \infty} \log \left(\frac{\left\langle\operatorname{Tr} W\left(a \vec{n}, a n_{t}\right)\right\rangle}{\left\langle\operatorname{Tr} W\left(a \vec{n}, a\left(n_{t}+1\right)\right)\right\rangle}\right)
$$

so that the potential at fixed $\vec{n}$ can be obtained by fitting to a constant the $\log$ in the rhs of Eq. (15) as a function of $n_{t}$, at least in a suitable stability range.

The application of such prescription in the present finite temperature context might seem not appropriate. Indeed, because of the limited Euclidean temporal extension, the static quark-antiquark potential is usually extracted from Polyakov loop correlators. However, on one hand such correlators turn out to be extremely noisy in our case, beyond the limit of feasibility, because of the relatively low temperatures considered in our investigation, which imply a large number of lattice sites in the temporal direction. On the other hand, because of the same reason, the temporal extension turns out be large enough $\left(N_{t}=40\right.$ in our particular case) and marginally compatible with an extraction of the potential also from Wilson loops. It is clear that one should be careful about possible systematic effects related to this compromise, however the results we are going to show are clear-cut enough to make such systematics less worrying.
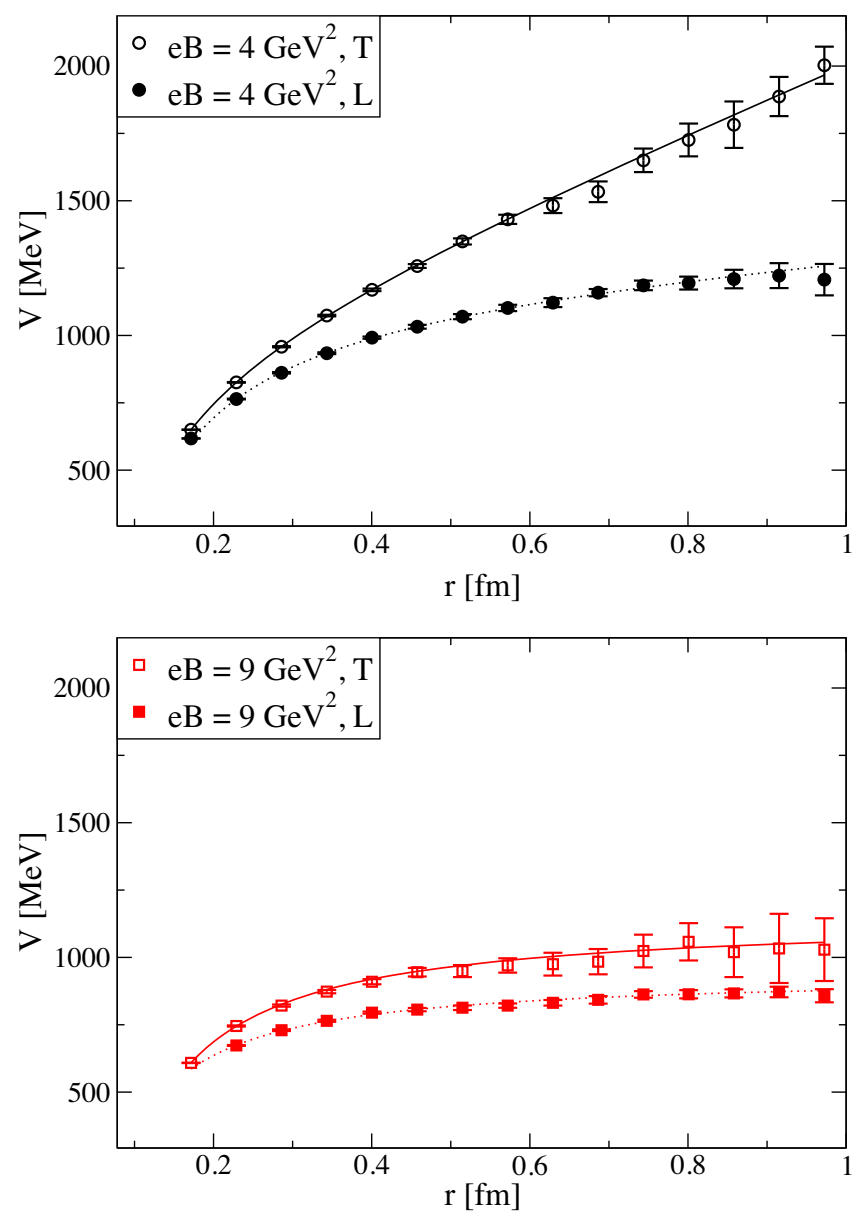

FIG. 8. Static quark-antiquark potential, extracted from Wilson loops computed on the $48^{3} \times 40$ lattice at the two different values of the magnetic field and separately for the transverse $(\mathrm{T})$ and longitudinal (L) directions. The reported curves correspond to fit to the Cornell potential (for $e B=4 \mathrm{GeV}^{2}$ ) or to a purely Coulombic potential (for $e B=9 \mathrm{GeV}^{2}$ ).

Results for the static quark-antiquark potential, computed for the two different orientations and magnetic fields, are shown in Fig. 8: the different behavior in the two phases is particularly clear, also by eye, for the transverse direction, where the linearly rising potential suddenly flattens moving from 4 to $9 \mathrm{GeV}^{2}$. In order to make a more quantitative analysis, we have tried to fit data according to the Cornell ansatz

$$
V(r)=V_{0}-\frac{\alpha}{r}+\sigma r
$$

obtaining the following results. For $e B=9 \mathrm{GeV}^{2}$, data are well fitted (with $\chi^{2} /$ d.o.f. $\lesssim 1$ ) by a purely Coulombic potential both in the transverse and in the longitudinal direction; if one tries to include a nonzero $\sigma$, the fit returns negative values (for $\sigma_{L}$ ) or values compatible with zero within errors (for $\sigma_{T}$ ). For $e B=4 \mathrm{GeV}^{2}$, instead, a nonzero string tension is clearly needed in the transverse direction, 
with $\sqrt{\sigma_{T}}=475(20) \mathrm{MeV}$, which is not far from the $T=$ 0 result obtained for the same lattice spacing in Ref. [23], $\sqrt{\sigma_{T}} \simeq 520 \mathrm{MeV}$; for the longitudinal direction a full fit to Eq. (16) returns $\sqrt{\sigma_{L}}=215(20) \mathrm{MeV}$ (which is close also in this case to the $T=0$ result $\sqrt{\sigma_{L}} \simeq 240 \mathrm{MeV}$ [23]); however one should consider that in this case reasonably good fits are obtained also assuming a purely Coulombic potential, if enough points are discarded at short distances.

To summarize, present evidence is compatible, within numerical uncertainties, with the transition from a strongly anisotropic confined phase to a completely deconfined phase, in which the string tension vanishes in all directions, as the critical line is crossed. Such evidence should be supported by future studies, aimed at assessing in a more precise way which string tension is vanishing or not on both sides of the transition. In this respects, several scenarios are plausible, including the possible existence of an intermediate phase in which $\sigma_{L}=0$ but $\sigma_{T} \neq 0$, for a subset of values of $B$ and $T$ (for predictions in this direction, see for instance Refs. [45,46]).

However, the sudden drop of the transverse string tension is a quite clear and undoubtful phenomenon even now. In the simplest scenario, one can assume that the critical temperature $T_{c}(B)$ continues its drop as a function of $B$ until it hits the ground at some critical magnetic field $B_{c}$. That would imply that, even at $T=0$, there is no transition to an anisotropically deconfined phase where $\sigma_{L}=0$ and $\sigma_{T} \neq 0$, but rather a sudden transition to a completely deconfined phase. Of course, even the assumption that $T_{c}(B)$ hits the ground is not supported, at the present time, by any other evidence.

\section{CONCLUSIONS AND PERSPECTIVES}

The numerical results presented in this study update our understanding of the QCD phase diagram in an external magnetic field in a substantial way, bringing new facts and new speculations into the overall picture. The main new results are that the (pseudo)critical temperature $T_{c}(B)$ continues its steady decrease as a function of $e B$, reaching values as a low as $60 \mathrm{MeV}$ for $e B$ of the order of $10 \mathrm{GeV}^{2}$, and that the crossover turns into a real first order transition for large enough magnetic fields. The latter fact has been speculated for a long time: in this paper we have provided first numerical evidence based on lattice simulation of $N_{f}=2+1$ QCD with physical quark masses. Moreover, we have provided a first rough location of the critical end point $\left(B_{E}, T_{E}\right)$ of the first order line, with $4 \mathrm{GeV}^{2}<e B_{E}<$ $9 \mathrm{GeV}^{2}$, or alternatively $65 \mathrm{MeV} \lesssim T_{E} \lesssim 95 \mathrm{MeV}$.

The existence and location of this critical end point have many significant implications: from a phenomenological point of view, especially for the possible consequences stemming from a strong first order cosmological QCD transition, which could be observable even nowadays $[21,22]$, and from a theoretical point of view, for a
$\mathrm{T}[\mathrm{MeV}]_{\wedge}$

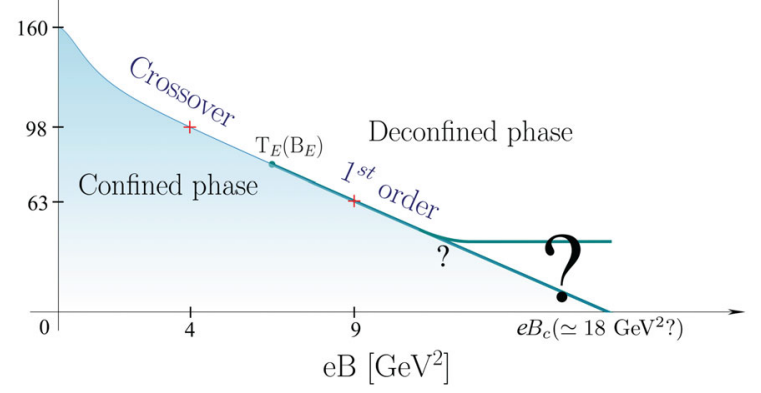

FIG. 9. Updated QCD phase diagram in an external magnetic field, based on new facts and new speculations emerging from our numerical investigations. The (pseudo)critical temperature $T_{c}(B)$ continues its steady drop as a function of $B$, and the transition switches from a crossover to first order at a critical end point located in the range $4 \mathrm{GeV}^{2}<e B_{E}<9 \mathrm{GeV}^{2}$ (or alternatively $\left.65 \mathrm{MeV}<T_{E}<95 \mathrm{MeV}\right)$. The fact that $T_{c}(B)$ hits the ground at some finite critical magnetic field $B_{c}$ or not remains an open question for future studies.

comparison with predictions from many effective model studies [47-54].

The new facts emerging from our investigation are reported in Fig. 9, which represents our present proposal for the QCD phase diagram. The proposal contains also some question marks, concerning open issues and speculations, that essentially regards the fate of $T_{c}(B)$ in the large $B$ limit. A naïve linear extrapolation of present determinations of $T_{c}$ in the $B-T$ plane would imply that $T_{c}$ vanishes for $e B_{c} \sim 20 \mathrm{GeV}^{2}$ : does that really happen, and in that case would $20 \mathrm{GeV}^{2}$ be a natural scale for $N_{f}=2+1$ QCD? Or does instead $T_{c}$ flatten for larger magnetic fields, approaching a finite value, or zero, only asymptotically?

The issue will be likely solved by future studies, and is strictly correlated to the fate of the confining properties of the QCD vacuum in a strong magnetic field. Indeed, if any critical magnetic field exists at $T=0$ where the confining properties of QCD get disrupted, this field likely coincides with the critical field where $T_{c}(B)$ hits the ground: results from Ref. [23] indicate that such a critical field, if any, is larger than $9 \mathrm{GeV}^{2}$, and so do the finite $T$ results presented here. One interesting point emerging from our study is that, as one crosses the critical line, the string tension seems to vanish, within our present numerical uncertainties, both in the longitudinal and in the transverse directions: if that applies down to $T=0$, then one should not expect any anisotropic deconfinement of the QCD vacuum at large fields, as hypothesized in Ref. [42], with the string tension vanishing only in the longitudinal direction, but rather a sudden quench of $\sigma$ in all directions at $B_{c}$.

There is a number of relevant issues that should be refined or investigated by future studies. First of all, one should consider that our study has been performed with a compromise between the need for a fine lattice spacing, in 
order to allow for a large magnetic field, and the need for large spatial sizes, in order to properly study thermodynamics. The compromise, given the presently available computational resources, has revealed to be not easy at all, essentially because of the unexpectedly low temperatures reached by the critical line, which forced us to work with low aspect ratios. Even if we have shown that systematics related to the finite UV cutoff and to the finite spatial size are reasonably under control, efforts should be pursued in the future to improve on such systematics: a first step in this direction would be the availability of larger spatial sizes in order to be able to study the transition at $e B=9 \mathrm{GeV}^{2}$ also on finer lattices.

A more precise location of the critical end point $\left(B_{E}, T_{E}\right)$ could be achieved following different approaches. Since the first order transition at $9 \mathrm{GeV}^{2}$ seems to be quite strong, one could consider lower values of $e B$ and investigate how the gap in physical observables changes along the transition line, trying to extrapolate the point where it vanishes. Alternatively, one could start from the low $B$ region, trying to detect the critical behavior associated with the end point, which is generally expected to be in the 3D-Ising universality class.

A future line of research should be dedicated to a precise characterization of the properties and differences of the two phases along the first order transition. In this investigation we have started a preliminary characterization of the confining properties, but many other relevant physical quantities should be considered, including a determination of the latent heat along the first order line and of the transport properties $[55,56]$ in both phases.

Finally, present results, in particular those regarding the critical end point, should be put in the framework of a more general and multidimensional view of the QCD phase diagram, including a finite baryon chemical potential [5763], different number of light fermions [64] or a finite rotation [65-68].

\section{ACKNOWLEDGMENTS}

We thank M. Cardinali for collaboration in the early stages of this study. Numerical simulations have been performed at the IT Center of the Pisa University and on the MARCONI and MARCONI100 machines at CINECA, based on the Project No. IscrB_QGPSMF and on the agreement between INFN and CINECA (under Projects No. INF20_npqcd, No. INF21_ npqcd). F. S. is supported by the Italian Ministry of University and Research (MUR) under Grant No. PRIN20172LNEEZ and by INFN under Grant No. GRANT73/CALAT.
[1] D. Kharzeev, K. Landsteiner, A. Schmitt, and H.-U. Yee, Lect. Notes Phys. 871, 1 (2013).

[2] J. O. Andersen, W. R. Naylor, and A. Tranberg, Rev. Mod. Phys. 88, 025001 (2016).

[3] V. A. Miransky and I. A. Shovkovy, Phys. Rep. 576, 1 (2015).

[4] V. Skokov, A. Y. Illarionov, and V. Toneev, Int. J. Mod. Phys. A 24, 5925 (2009).

[5] V. Voronyuk, V. D. Toneev, W. Cassing, E. L. Bratkovskaya, V. P. Konchakovski, and S. A. Voloshin, Phys. Rev. C 83, 054911 (2011).

[6] A. Bzdak and V. Skokov, Phys. Lett. B 710, 171 (2012).

[7] W.-T. Deng and X.-G. Huang, Phys. Rev. C 85, 044907 (2012).

[8] K. Tuchin, Adv. High Energy Phys. 2013, 490495 (2013).

[9] R. Holliday, R. McCarty, B. Peroutka, and K. Tuchin, Nucl. Phys. A957, 406 (2017).

[10] R. C. Duncan and C. Thompson, Astrophys. J. 392, L9 (1992).

[11] T. Vachaspati, Phys. Lett. B 265, 258 (1991).

[12] D. Grasso and H. R. Rubinstein, Phys. Rep. 348, 163 (2001).

[13] M. D’Elia, S. Mukherjee, and F. Sanfilippo, Phys. Rev. D 82, 051501 (2010).

[14] E. M. Ilgenfritz, M. Kalinowski, M. Muller-Preussker, B. Petersson, and A. Schreiber, Phys. Rev. D 85, 114504 (2012).
[15] G. S. Bali, F. Bruckmann, G. Endrodi, Z. Fodor, S. D. Katz, S. Krieg, A. Schafer, and K. K. Szabo, J. High Energy Phys. 02 (2012) 044.

[16] V. G. Bornyakov, P. V. Buividovich, N. Cundy, O. A. Kochetkov, and A. Schäfer, Phys. Rev. D 90, 034501 (2014).

[17] H. T. Ding, C. Schmidt, A. Tomiya, and X. D. Wang, Phys. Rev. D 102, 054505 (2020).

[18] M. D’Elia, F. Manigrasso, F. Negro, and F. Sanfilippo, Phys. Rev. D 98, 054509 (2018).

[19] G. Endrodi, M. Giordano, S. D. Katz, T. G. Kovács, and F. Pittler, J. High Energy Phys. 07 (2019) 007.

[20] G. Endrodi, J. High Energy Phys. 07 (2015) 173.

[21] E. Witten, Phys. Rev. D 30, 272 (1984).

[22] J. H. Applegate and C. J. Hogan, Phys. Rev. D 31, 3037 (1985).

[23] M. D’Elia, L. Maio, F. Sanfilippo, and A. Stanzione, Phys. Rev. D 104, 114512 (2021).

[24] P. Weisz, Nucl. Phys. B212, 1 (1983).

[25] G. Curci, P. Menotti, and G. Paffuti, Phys. Lett. 130B, 205 (1983); 135B, 516(E) (1984).

[26] J. Kogut and L. Susskind, Phys. Rev. D 11, 395 (1975).

[27] C. Morningstar and M. J. Peardon, Phys. Rev. D 69, 054501 (2004).

[28] Y. Aoki, S. Borsanyi, S. Durr, Z. Fodor, S. D. Katz, S. Krieg, and K. K. Szabo, J. High Energy Phys. 06 (2009) 088. 
[29] S. Borsanyi, G. Endrodi, Z. Fodor, A. Jakovac, S. D. Katz, S. Krieg, C. Ratti, and K. K. Szabo, J. High Energy Phys. 11 (2010) 077.

[30] S. Borsanyi, Z. Fodor, C. Hoelbling, S. D. Katz, S. Krieg, and K. K. Szabo, Phys. Lett. B 730, 99 (2014).

[31] G. 't Hooft, Nucl. Phys. B153, 141 (1979).

[32] M. H. Al-Hashimi and U. J. Wiese, Ann. Phys. (Amsterdam) 324, 343 (2009).

[33] M. D’Elia, Lect. Notes Phys. 871, 181 (2013).

[34] G. Endrodi, Z. Fodor, S. D. Katz, and K. K. Szabo, J. High Energy Phys. 04 (2011) 001.

[35] C. Bonati, E. Calore, S. Coscetti, M. D’Elia, M. Mesiti, F. Negro, S. F. Schifano, G. Silvi, and R. Tripiccione, Int. J. Mod. Phys. C 28, 1750063 (2017).

[36] C. Bonati, E. Calore, M. D’Elia, M. Mesiti, F. Negro, F. Sanfilippo, S. F. Schifano, G. Silvi, and R. Tripiccione, Int. J. Mod. Phys. C 29, 1850010 (2018).

[37] H. T. Ding, S. T. Li, Q. Shi, A. Tomiya, X. D. Wang, and Y. Zhang, Acta Phys. Pol. B Proc. Suppl. 14, 403 (2021).

[38] V. A. Miransky and I. A. Shovkovy, Phys. Rev. D 66, 045006 (2002).

[39] A. J. Mizher, M. N. Chernodub, and E. S. Fraga, Phys. Rev. D 82, 105016 (2010).

[40] C. Bonati, M. D'Elia, and A. Rucci, Phys. Rev. D 92, 054014 (2015).

[41] C. Bonati, M. D'Elia, M. Mariti, M. Mesiti, F. Negro, and F. Sanfilippo, Phys. Rev. D 89, 114502 (2014).

[42] C. Bonati, M. D’Elia, M. Mariti, M. Mesiti, F. Negro, A. Rucci, and F. Sanfilippo, Phys. Rev. D 94, 094007 (2016).

[43] C. Bonati, S. Calì, M. D’Elia, M. Mesiti, F. Negro, A. Rucci, and F. Sanfilippo, Phys. Rev. D 98, 054501 (2018).

[44] C. Bonati, M. D'Elia, M. Mariti, M. Mesiti, F. Negro, A. Rucci, and F. Sanfilippo, Phys. Rev. D 95, 074515 (2017).

[45] M. N. Chernodub, Mod. Phys. Lett. A 29, 1450162 (2014).

[46] M. N. Chernodub, Phys. Rev. D 82, 085011 (2010).

[47] T. D. Cohen and N. Yamamoto, Phys. Rev. D 89, 054029 (2014).
[48] J. Moreira, P. Costa, and T. E. Restrepo, Eur. Phys. J. A 57, 123 (2021).

[49] A. Ayala, L. A. Hernández, M. Loewe, and C. Villavicencio, Eur. Phys. J. A 57, 234 (2021).

[50] S. S. Avancini, D. P. Menezes, M. B. Pinto, and C. Providencia, Phys. Rev. D 85, 091901 (2012).

[51] P. Costa, M. Ferreira, H. Hansen, D. P. Menezes, and C. Providência, Phys. Rev. D 89, 056013 (2014).

[52] A. Bandyopadhyay and R. L. S. Farias, Eur. Phys. J. Special Topics 230, 719 (2021).

[53] J. O. Andersen, Eur. Phys. J. A 57, 189 (2021).

[54] N. Mueller and J. M. Pawlowski, Phys. Rev. D 91, 116010 (2015).

[55] N. Astrakhantsev, V. V. Braguta, M. D’Elia, A. Y. Kotov, A. A. Nikolaev, and F. Sanfilippo, Phys. Rev. D 102, 054516 (2020).

[56] S. I. Finazzo, R. Critelli, R. Rougemont, and J. Noronha, Phys. Rev. D 94, 054020 (2016); 96, 019903(E) (2017).

[57] V. V. Braguta, M. N. Chernodub, A. Y. Kotov, A. V. Molochkov, and A. A. Nikolaev, Phys. Rev. D 100, 114503 (2019).

[58] M. Szymański, Eur. Phys. J. Special Topics 229, 3387 (2020).

[59] P. V. Buividovich, D. Smith, and L. von Smekal, Eur. Phys. J. A 57, 293 (2021).

[60] R. A. Abramchuk, M. A. Andreichikov, Z. V. Khaidukov, and Y. A. Simonov, Eur. Phys. J. C 79, 1040 (2019).

[61] A. Ayala, C. A. Dominguez, L. A. Hernandez, M. Loewe, and R. Zamora, Phys. Rev. D 92, 096011 (2015).

[62] E. J. Ferrer and V. de la Incera, Eur. Phys. J. A 52, 266 (2016).

[63] V. Skokov, Phys. Rev. D 85, 034026 (2012).

[64] M. Kawaguchi, S. Matsuzaki, and A. Tomiya, J. High Energy Phys. 12 (2021) 175.

[65] A. Yamamoto and Y. Hirono, Phys. Rev. Lett. 111, 081601 (2013).

[66] K. Fukushima, Prog. Part. Nucl. Phys. 107, 167 (2019).

[67] H. L. Chen, X. G. Huang, and J. Liao, Lect. Notes Phys. 987, 349 (2021).

[68] A. Yamamoto, Eur. Phys. J. A 57, 211 (2021). 